\title{
Tackling hearing loss to improve the care of older
} adults

The authors' affiliations in this Analysis (BMJ 2018;360:k21, doi:10.1136/bmj.k21) contain two errors. Joshua Chodosh is the Freedman professor of geriatric medicine, and Barbara
Weinstein is at the City University of New York. The original PDF of this article was missing the footnotes in table 1; this has now been corrected. 